\title{
Potencial de produção de sementes de gramíneas para a implantação em gramados ${ }^{(1)}$
}

\author{
JOSIANE VARGAS DE OLIVEIRA MAXIMINO(2)*, MARCO AURÉLIO SCHIAVON MACHADO(2), \\ ANDRÉA MITTELMANN(3), ELIÉZER DA CUNHA PINHEIRO(4), ELIZANDRO DA SILVA PIRES ${ }^{(4)}$, MIKAEL BUENO \\ LONGARAY(5), FRANCISCO HUMBERTO DÜBBERN DE SOUZA(6) e ELISABETH REGINA TEMPEL STUMPF(7)
}

\section{RESUMO}

Os gêneros Paspalum e Axonopus encontram-se entre as principais gramíneas de estação quente utilizadas para gramados. A propagação via sementes contribui para diminuir o custo de implantação, além de manter as características exatas do genótipo da planta mãe, por se tratar de espécies apomíticas. O objetivo deste trabalho foi avaliar o potencial de produção de sementes de dezessete acessos de gramíneas das espécies Paspalum notatum, P. lepton, P. lividum e Axonopus parodii. O experimento foi conduzido no município de Capão do Leão, Rio Grande do Sul, em blocos casualizados, com quatro repetições. As variáveis avaliadas foram: número de inflorescências por área, número de flósculos por inflorescência e potencial de produção de sementes (PPS). Para mensurar o potencial de produção de sementes dos acessos propõe-se a equação: PPS $=\mathrm{n}^{\circ}$ de flósculos por inflorescência $\mathrm{x} \mathrm{n}^{\mathrm{o}}$ de inflorescências por $\mathrm{m}^{2}$. Houve efeito de ano, acesso e interação entre anos e acessos para número de inflorescências por área e potencial de produção de sementes. Para a variável número de flósculos por inflorescência, não houve efeito de ano. A produção potencial da safra 2013/2014 variou de 19152,00 a 135062,70 sementes $\mathrm{m}^{-2}$, destacando-se o acesso PN 09 da espécie $P$. notatum e na safra 2014/2015 a produção potencial variou de 9973,75 a 81536,75 sementes m² $^{-2}$, destacando-se o acesso PN 11 da espécie P. notatum. Os acessos PN 11, PN 09, PN 10 e AP 01 estiveram no terço superior no ranking de produtividade potencial nas duas safras, e a grama-batatais no terço inferior. Existe interação genótipo-ambiente para todas as características avaliadas. Entretanto, existem acessos que apresentam produção potencial de sementes consistentemente superior à testemunha grama-batatais, tendo maior potencial para a exploração na implantação de gramados por sementes.

Palavras-chave: Paspalum notatum, P. lepton, P. lividum, Axonopus parodii, inflorescência, apomixia.

\begin{abstract}
Potential of grass seed production for new lawns

The Paspalum and Axonopus genera are among the main warm season grasses used for lawns. The seed propagation contributes to the decrease of the cost of establishment, besides maintaining the exact characteristics of the mother plant genotype, because they are apomictic species. The objective of this work was to evaluate the seed production potential of seventeen grass accesses of the species Paspalum notatum, P. lepton, P. lividum and Axonopus parodii. The experiment was conducted at Capão do Leão, Rio Grande do Sul State, Brazil, in a randomized block design, with four replications. The evaluated variables were: number of inflorescences per area, number of florets per inflorescence and seed production potential (SPP). In order to measure the seed production potential of the accesses, the equation proposed is: SPP = number of florets per inflorescence $\mathrm{x}$ number of inflorescences per $\mathrm{m}^{2}$. There were year, access and interaction between years and accesses effect for the traits number of inflorescences per area and seed production potential. For the number of florets per inflorescence, there was no year effect. Potential production for the 2013/2014 harvest, ranged from $19,152.00$ to $135,062.70$ seeds $\mathrm{m}^{-2}$, with PN 09 of the P. notatum species standing out. In the 2014/2015 harvest, the seed production potential ranged from 9,973.75 to 81,536.75 seeds $\mathrm{m}^{-2}$, highlighting the access PN 11 of the species P. notatum. The accesses PN 11, PN 09, PN 10 and AP 01 were in the top third of the seed production potential ranking in the two harvests, and "grama-batatais" was in the lower third. There is genotype-environment interaction for all characteristics evaluated. However, there are accesses that show seed production potential consistently superior to the "grama-batatais" control, and have a greater potential for exploitation in the establishment of lawns by seeds.
\end{abstract}

Keywords: Paspalum notatum, P. lepton, P. lividum, Axonopus parodii, inflorescence, apomixy.

DOI: http://dx.doi.org/10.14295/oh.v23i2.1011

(1) Recebido em 08/03/2017 e aceito em 20/06/2017

(2) Universidade Federal de Pelotas (UFPel), Faculdade de Agronomia, Pelotas-RS, Brasil. *Autor correspondente: josianemaximino@gmail.com

${ }^{(3)}$ Embrapa Gado de Leite, Embrapa Clima Temperado, Juiz de Fora-MG, Brasil.

(4) Universidade Federal de Pelotas (UFPel), Pelotas-RS, Brasil.

${ }^{(5)}$ Embrapa Clima Temperado, Pelotas-RS, Brasil.

(6) Embrapa Pecuária Sudeste, São Carlos-SP, Brasil.

(7) Instituto Federal Sul-rio-grandense, Pelotas-RS, Brasil.

Licensed by CC BY 4.0 


\section{INTRODUÇÃO}

As gramíneas são de extrema importância ecológica, pois impedem a erosão, auxiliam na fixação do solo, uma vez que tem raízes, estolões e rizomas que formam uma rede sob a superfície e, ainda, não só auxiliam na fixação, mas alguns deles são o primeiro passo para a sua formação (PÉREZ-ARBELÁEZ, 1978). Muitas espécies de gramíneas podem ser utilizadas para restaurar solos degradados (GOULD e SHAW, 1992).

Algumas cultivares de grama são conhecidas como fonte de alimento para animais, com ótimo potencial forrageiro, mas é possível utilizá-las como cobertura vegetal para a fixação de dunas, em contenção da erosão nas encostas de rodovias, em parques, para fins ornamentais e em projetos paisagísticos, auxiliando na diversificação das paisagens.

As gramíneas de estação quente utilizadas para gramados são principalmente dos gêneros Axonopus, Cynodon, Eremochloa, Paspalum, Stenotaphrum e Zoysia (BUSEY, 1989; LÓPEZ et al., 2013). Os gêneros Axonopus e Paspalum são morfologicamente parecidos, sendo diferenciados pela orientação das espiguetas: solitárias, subsésseis e adaxiais em Axonopus e espiguetas abaxiais, pediceladas e solitárias ou pareadas em Paspalum (MORRONE et al., 2012).

Paspalum é um dos gêneros de Paniceae de maior importância econômica, seja pela qualidade de forragem que produzem suas espécies ou por incluir táxons empregados na alimentação ou na medicina popular (ZULOAGA e MORRONE, 2005). Este gênero compreende espécies distribuídas em regiões tropicais, subtropicais e temperadas, estas principalmente nos Estados Unidos, com poucas espécies que habitam o Velho Mundo (CLAYTON e RENVOIZE, 1986). É muitas vezes dominante em lugares abertos, como pântanos, savanas e pastos e encontrado também em ambientes de selva ou floresta e dunas costeiras ou em áreas alagados. Possui adaptação a diferentes estresses ambientais (DUNCAN, 2003). Várias espécies se comportam como ervas daninhas, sendo encontradas em beiras de estradas, estradas de ferro e solos modificados (ZULOAGA e MORRONE, 2005).

No Brasil, o gênero Paspalum engloba o maior número de espécies de gramíneas nativas. O Sul das Américas é o centro de origem e de diversificação genética das espécies desse gênero, que compreende cerca de 400 espécies, das quais 130 ocorrem no Brasil. As espécies e os ecótipos estão distribuídos principalmente nas regiões Centro-Sul do Brasil, Leste da Bolívia, Norte da Argentina, Paraguai e Uruguai (BATISTA, 1998). A espécie mais popular, a grama-batatais (Paspalum notatum), é a mais comum na formação dos campos nativos do Rio Grande do Sul (MOHRDIECK, 1993). Esta espécie é de grande utilização contra a erosão, pois suas raízes se entrelaçam, cobrindo o terreno e retendo o solo, sendo cultivada, com essa finalidade, em terrenos acidentados e em taludes ao longo de canais e rodovias (KISSMANN, 1999).

Axonopus é um gênero neotropical representado por cerca de 100 espécies com ampla distribuição em regiões tropicais e subtropicais da América e com poucas espécies ocorrendo também na Ásia, África, Austrália e Ilhas do Pacífico (GIUSSANI et al., 2001). No Brasil, o gênero está representado por cerca de 80 espécies; sendo que, em função de sua extensão e posição geográfica, é o país que apresenta maior riqueza de espécies (GIRALDO-CANÃS e MAYORGA, 2001). A espécie Axonopus parodii é morfologicamente descrita como perene, estolonífera, com rizomas supraterrâneos (semelhantes aos de Paspalum notatum), estolhos vigorosos, cujo aspecto lembra Stenotaphrum secundatum e colmos floríferos com 30 a 60 $\mathrm{cm}$ de altura (HICKENBICK et al., 1975).

Os gramados podem ser implantados de duas formas: por meio da propagação vegetativa ou através de sementes. O gramado formado por meio de propágulos vegetativos apresenta bom desenvolvimento inicial, porém existem várias limitações, dentre elas os altos custos de implantação, a necessidade de grande quantidade de material de propagação e de mão-de-obra para implantação em grandes áreas e a disseminação de pragas e doenças, além de limitações relacionadas ao transporte e acondicionamento, por tratar-se de material perecível. Atualmente, no Brasil, o número de espécies utilizadas para a formação de gramados é pequeno, e a maioria dos propágulos utilizados para esta finalidade são oriundos de áreas nativas, o que acaba contribuindo com a degradação do solo, prejudicando a reutilização da área da qual são retirados (SOUZA, 2013b).

Segundo Souza (2013b), a propagação via sementes pode contribuir para a redução dos custos de formação de gramados. Por se tratar de espécies apomíticas, as sementes permitem a reprodução exata do genótipo materno.

A prática de implantação de gramados por sementes no Brasil começou nos anos 80 por meio de sementes de Axonopus sp. e Paspalum notatum, geralmente vindas da Austrália. Por apresentarem germinação muito lenta, em torno de 60 dias, a formação de gramados por meio de sementes caiu em descrédito e durante algum tempo não foi mais utilizada. Na Europa e Estados Unidos, no entanto, a formação de gramados por meio de sementes é uma prática, sendo o método preferido para a formação de pastagens, em especial onde há pouca disponibilidade de mão-de-obra ou onde as áreas a serem cultivadas são extensas, sendo a amplitude da utilização das cultivares de gramíneas, nessas condições, dependente da disponibilidade de sementes (SOUZA, 2013c). Segundo Pizarro (2000), o que tem limitado a multiplicação comercial através de sementes no gênero Paspalum é a falta de estudos sobre produção de sementes.

Desde 2011 a Embrapa tem avaliado e selecionado acessos de gramíneas com características funcionais para gramados. Após as avaliações relacionadas ao desenvolvimento das espécies, à eficiência de cobertura do solo, resistência à geada e competitividade com espécies invasoras, faz-se necessário avaliar a produção de sementes, com o intuito de analisar se a propagação via semente é uma alternativa para os acessos selecionados. Assim sendo, o objetivo deste trabalho foi avaliar o potencial de produção de sementes de dezessete acessos de gramíneas das espécies Paspalum notatum, P. lepton, P. lividum e Axonopus parodii. 


\section{MATERIAL E MÉTODOS}

O experimento foi conduzido na Estação Experimental Terras Baixas da Embrapa Clima Temperado, no município de Capão do Leão, Rio Grande do Sul (31'52'00" S, $52^{\circ} 21^{\prime} 24$ ” W, altitude 13,24 m), nas safras de verão 2013/2014 e 2014/2015.

Tabela 1. Produção potencial de sementes de 17 acessos dos gêneros Paspalum e Axonopus na safra 2013/2014. Capão do Leão-RS, Brasil.

Table 1. Seed production potential of 17 accesses of Paspalum and Axonopus genus, in the 2013/2014 crop. Capão do Leão-RS, Brazil.

\begin{tabular}{|c|c|c|c|c|}
\hline Acesso & Espécie & $\underset{\left(n^{0} m^{-2}\right)}{\text { NIA }}$ & $\begin{array}{l}\text { NFI } \\
\left(\mathrm{n}^{0}\right)\end{array}$ & $\begin{array}{c}\text { PPS } \\
\left(\mathrm{n}^{\mathbf{0}} \text { sementes } \mathrm{m}^{-2}\right)\end{array}$ \\
\hline PN 09 & P. notatum & $338,00^{a^{*}}$ & $179,70^{a}$ & $135.062,70^{\mathrm{a}}$ \\
\hline AP 01 & Axonopus parodii & $508,75^{\text {a }}$ & $85,10^{\mathrm{e}}$ & $98.430,70^{\mathrm{b}}$ \\
\hline PN 10 & P. notatum & $441,50^{\text {a }}$ & $169,17^{\text {a }}$ & $93.218,70^{\mathrm{b}}$ \\
\hline PN 11 & P. notatum & $520,75^{\text {a }}$ & $99,65^{d}$ & $82.864,45^{b}$ \\
\hline PN 07 & P. notatum & $636,25^{a}$ & $117,90^{\mathrm{c}}$ & $73.341,25^{b}$ \\
\hline PN 06 & P. notatum & $780,25^{\text {a }}$ & $103,20^{d}$ & $58.257,72^{\mathrm{c}}$ \\
\hline PN 12 & P. notatum & $395,25^{\text {a }}$ & $134,97^{\mathrm{b}}$ & $54.314,25^{\mathrm{c}}$ \\
\hline PN 13 & P. notatum & $338,66^{a}$ & $84,10^{\mathrm{e}}$ & $50.695,13^{c}$ \\
\hline PN 02 & P. notatum & $422,25^{a}$ & $136,77^{b}$ & $50.064,40^{c}$ \\
\hline PL 03 & P. lepton & $595,25^{\mathrm{a}}$ & $86,50^{\text {e }}$ & $46.188,15^{\mathrm{c}}$ \\
\hline PL 01 & P. lepton & $687,50^{\mathrm{a}}$ & $76,40^{\mathrm{f}}$ & $44.854,45^{c}$ \\
\hline PN 01 & P. notatum & $313,25^{\text {a }}$ & $89,25^{\mathrm{e}}$ & $42.173,90^{\mathrm{c}}$ \\
\hline PN 03 & P. notatum & $557,75^{a}$ & $92,22^{\mathrm{e}}$ & $34.439,92^{d}$ \\
\hline PL 02 & P. lividum & $599.66^{\text {a }}$ & $66,73^{f}$ & $34.030,13^{d}$ \\
\hline PN 05 & P. notatum & $424,75^{\text {a }}$ & $84,80^{\text {e }}$ & $27.852,90^{d}$ \\
\hline Testemunha & P. notatum & $448,75^{\text {a }}$ & $98,77^{\mathrm{d}}$ & $26.583,15^{d}$ \\
\hline PN 14 & P. notatum & $540,00^{a}$ & $139,00^{\mathrm{b}}$ & $24.881,00^{\mathrm{d}}$ \\
\hline PN 04 & P. notatum & $359,75^{\text {a }}$ & $112,75^{c}$ & $19.152,00^{d}$ \\
\hline
\end{tabular}

Médias seguidas de mesma letra não diferem significativamente pelo teste de Scott-Knot com $a=0,05$. Número de inflorescências por área (NIA), número de flósculos por inflorescência (NFS) e produção potencial de sementes (PPS). Means followed by the same letter did not differ significantly by the Scott-Knot test with (a=0.05). Number of inflorescences per area (NIA), number of florets per inflorescence (NFS) and potential seed production (PPS). 
Tabela 2. Produção potencial de sementes de 17 acessos dos gêneros Paspalum e Axonopus na safra 2014/2015. Capão do Leão-RS, Brasil.

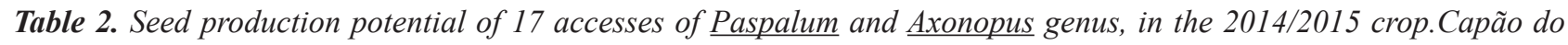
Leão-RS, Brazil.

\begin{tabular}{|c|c|c|c|c|}
\hline Acesso & Espécie & $\begin{array}{c}\text { NIA } \\
\left(\mathbf{n}^{\mathbf{0}} / \mathbf{m}^{\mathbf{2}} \mathbf{)}\right.\end{array}$ & $\begin{array}{c}\text { NFI } \\
\mathbf{( n}^{\mathbf{0}} \mathbf{c}\end{array}$ & $\begin{array}{c}\text { PPS } \\
\left(\mathbf{n}^{\mathbf{0}} \mathbf{s e m e n t e s} / \mathbf{m}^{\mathbf{2}}\right)\end{array}$ \\
\hline PN 09 & P. notatum & $301,50^{\mathrm{b}}$ & $148,80^{\mathrm{b}}$ & $44.938,20^{\mathrm{b}}$ \\
\hline AP 01 & Axonopus parodii & $349,50^{\mathrm{b}}$ & $112,46^{\mathrm{c}}$ & $43.782,20^{\mathrm{b}}$ \\
\hline PN 10 & P. notatum & $316,00^{\mathrm{b}}$ & $173,40^{\mathrm{a}}$ & $43.904,10^{\mathrm{b}}$ \\
\hline PN 11 & P. notatum & $793,50^{\mathrm{a}}$ & $103,40^{\mathrm{c}}$ & $81.536,70^{\mathrm{a}}$ \\
\hline PN 07 & P. notatum & $226,50^{\mathrm{b}}$ & $110,15^{\mathrm{c}}$ & $27.070,30^{\mathrm{c}}$ \\
\hline PN 06 & P. notatum & $426,50^{\mathrm{a}}$ & $100,06^{\mathrm{c}}$ & $40.479,50^{\mathrm{b}}$ \\
\hline PN 12 & P. notatum & $106,00^{\mathrm{c}}$ & $136,05^{\mathrm{b}}$ & $25.073,20^{\mathrm{c}}$ \\
\hline PN 13 & P. notatum & $505,33^{\mathrm{a}}$ & $81,50^{\mathrm{d}}$ & $34.926,40^{\mathrm{b}}$ \\
\hline PN 02 & P. notatum & $124,50^{\mathrm{c}}$ & $144,75^{\mathrm{b}}$ & $18.039,90^{\mathrm{c}}$ \\
\hline PL 03 & P. lepton & $551,50^{\mathrm{a}}$ & $79,90^{\mathrm{d}}$ & $43.403,20^{\mathrm{b}}$ \\
\hline PL 01 & P. lepton & $667,00^{\mathrm{a}}$ & $77,50^{\mathrm{d}}$ & $52.198,00^{\mathrm{b}}$ \\
\hline PN 01 & P. notatum & $334,00^{\mathrm{b}}$ & $95,60^{\mathrm{c}}$ & $31.876,40^{\mathrm{b}}$ \\
\hline PN 03 & P. notatum & $250,00^{\mathrm{b}}$ & $116,30^{\mathrm{c}}$ & $28.527,60^{\mathrm{c}}$ \\
\hline PL 02 & P. lividum & $560,00^{\mathrm{a}}$ & $95,93^{\mathrm{c}}$ & $52.902,13^{\mathrm{b}}$ \\
\hline PN 05 & P. notatum & $247,50^{\mathrm{b}}$ & $101,60^{\mathrm{c}}$ & $24.509,60^{\mathrm{c}}$ \\
\hline Testemunha & P. notatum & $274,00^{\mathrm{b}}$ & $89,46^{\mathrm{d}}$ & $25.188,93^{\mathrm{c}}$ \\
\hline PN 14 & P. notatum & $270,00^{\mathrm{b}}$ & $105,90^{\mathrm{c}}$ & $16.814,20^{\mathrm{c}}$ \\
\hline PN 04 & P. notatum & $89,50^{\mathrm{c}}$ & $110,30^{\mathrm{c}}$ & $9.973,60^{\mathrm{d}}$ \\
\hline
\end{tabular}

Médias seguidas de mesma letra não diferem significativamente pelo teste de Scott-Knot $(a=0,05)$. Número de inflorescências por área (NIA), número de flósculos por inflorescência (NFS) e produção potencial de sementes (PPS). Means followed by the same letter did not differ significantly by the Scott-Knot test with ( $a=0.05)$. Number of inflorescences per area (NIA), number of florets per inflorescence (NFS) and potential seed production (PPS).

O solo da região é um Planossolo Háplico Eutrófico típico. Com base nos resultados de análise de solo (teores de argila $11 \%$; matéria orgânica $0,9 \%$; pH 5,2; fósforo 68,2 e potássio $41 \mathrm{mg} \cdot \mathrm{dm}^{-3}$; saturação de bases $41 \%$ e CTC efetiva 2,1), foi realizada correção e adubação conforme recomendações (SOCIEDADE BRASILEIRA DE CIÊNCIA DO SOLO, 2004). Em 6 de dezembro de 2011 as mudas do tipo sprig, propágulos vegetativos individuais de raiz nua, ou seja, partes de rizoma ou estolões, provenientes da Embrapa Pecuária Sudeste, foram transplantadas para as parcelas experimentais de $6 \mathrm{~m}^{2}$, em um delineamento de blocos casualizados, com quatro repetições. O plantio foi realizado em linhas, com espaçamento de $0,25 \mathrm{~m}$ entre linhas e de $0,15 \mathrm{~m}$ entre mudas na linha. Após dois anos, iniciou-se a avaliação sobre a produção potencial de sementes dos acessos.

Em setembro de 2013 foi realizada adubação de reposição com $300 \mathrm{Kg} \mathrm{ha-1}$ de N-P-K (5-20-20) e 40 $\mathrm{kg} \mathrm{ha} \mathrm{H}^{-1}$ de nitrogênio na forma uréia e em outubro foi realizado o emparelhamento das parcelas a uma altura de corte de $5 \mathrm{~cm}$ da superfície do solo (LÓPEZ et al., 2013). Os procedimentos foram repetidos na primavera de 2014 .

As variáveis avaliadas foram: número de inflorescências por área (NIA), contado em duas subamostras de $0,25 \mathrm{~m}^{2}$ por parcela e corrigido para $1 \mathrm{~m}^{2}$; número de flósculos por inflorescência (NFI), contado em vinte inflorescências por parcela; potencial de produção de sementes (PPS), obtido pela seguinte equação: $\operatorname{PPS}=\mathrm{n}^{\circ}$ de flósculos por inflorescência $\mathrm{x} \mathrm{n}^{\circ}$. de inflorescências por $\mathrm{m}^{2}$.

A análise estatística compreendeu, para cada característica avaliada, as análises de variância por safra e a conjunta, incluindo os efeitos de safra, acesso e da interação entre acessos e safras. A comparação entre as médias dos acessos foi realizada pelo teste de ScottKnott a $5 \%$ de probabilidade e a comparação entre as médias das safras foi realizada através do teste $\mathrm{F}$ a $5 \%$ de probabilidade.

\section{RESULTADOS E DISCUSSÃO}

Houve efeito significativo de safra, acesso e interação entre safras e acessos para as variáveis número de inflorescências por área e potencial de produção de sementes. Para a variável número de flósculos por inflorescência, apenas os efeitos de acesso e da interação entre safras e acessos foram significativos. Houve maior número de inflorescências $/ \mathrm{m}^{2}$ na safra de 2013/2014, a qual também apresentou maior potencial de produção de sementes (Tabela 3). 
Tabela 3. Médias das safras 2013/2014 e 2014/2015 para os caracteres número de inflorescências por área (NIA), número de flósculos por inflorescência (NFS) e produção potencial de sementes (PPS). Capão do Leão-RS, Brasil.

Table 3. Averages of the 2013/2014 and 2014/2015 crops for the traits number of inflorescences per area, number of florets per inflorescence and seed production potential.Capão do Leão-RS, Brazil.

\begin{tabular}{|c|c|c|c|}
\hline Safra & $\underset{\left(n^{0} \mathbf{m}^{-2}\right)}{\text { NIA }}$ & $\begin{array}{l}\text { NFS } \\
\left(n^{0}\right)\end{array}$ & $\begin{array}{c}\text { PPS } \\
\left(\mathrm{n}^{\mathbf{0}} \text { sementes } \mathrm{m}^{-2}\right)\end{array}$ \\
\hline $2013 / 2014$ & $507,07^{\mathrm{A}}$ & $107,06^{\mathrm{A}}$ & $56383^{\mathrm{A}}$ \\
\hline $2014 / 2015$ & $338,25^{\text {B }}$ & $110,57^{\mathrm{A}}$ & $35570^{\mathrm{B}}$ \\
\hline
\end{tabular}

Efeito de safra testado pelo teste F com $(a=0,05)$. Crop effect tested with $F$ test $(a=0,05)$.

Segundo Carambula (1981), os componentes da produção de sementes são determinados desde o desenvolvimento vegetativo até as etapas do desenvolvimento reprodutivo, destacando-se o número de inflorescências, o número de flores por inflorescência, a porcentagem de germinação e o peso das sementes. No presente trabalho, duas destas características foram avaliadas. Portanto, é necessário levar em consideração as condições ambientais durante todo o período de cultivo. Tais condições devem ser favoráveis ao desenvolvimento da espécie e à concepção das sementes. Estresses abióticos estão relacionados com o ambiente e são considerados um dos principais fatores limitantes nos cultivos agrícolas, pois incidem no crescimento e na produção das plantas, desencadeando uma série de transtornos morfológicos, bioquímicos e moleculares (LÓPEZ et al., 2013). Embora na média dos meses as duas safras não tenham diferido de forma importante para os principais fatores climáticos (Tabela 4), variações na distribuição ao longo do período podem ter contribuído para as variações entre as duas safras avaliadas. Ambas tiveram precipitações acima das normais climáticas.

Tabela 4. Temperaturas médias, mínimas e máximas, radiação solar, precipitação e número de dias de precipitação mensais referentes às safras de verão 2013/2014 e 2014/2015. Capão do Leão-RS, Brasil.

Table 4. Monthly mean, minimal and maximum temperatures, solar radiation, rainfall, and number of days of raining referring to 2013/2014 and 2014/2015 summer crops. Capão do Leão-RS, Brazil

\begin{tabular}{|c|c|c|c|c|c|c|}
\hline Safra/Mês & $\begin{array}{c}\text { Temperatura } \\
\text { Média } \\
\left({ }^{\circ} \mathbf{C}\right)\end{array}$ & $\begin{array}{c}\text { Temperatura } \\
\text { Média das } \\
\text { Mínimas }\left({ }^{\circ} \mathrm{C}\right)\end{array}$ & $\begin{array}{c}\text { Temperatura } \\
\text { Média das } \\
\text { Máximas ( }{ }^{\circ} \mathrm{C} \text { ) }\end{array}$ & $\begin{array}{l}\text { Radiação Solar } \\
\left(\text { Cal cm }^{-2} \text { dia }^{-1}\right)\end{array}$ & $\begin{array}{c}\text { Precipitação } \\
(\mathbf{m m})\end{array}$ & $\begin{array}{c}\text { Dias de } \\
\text { Precipitação } \\
\text { (No.) }\end{array}$ \\
\hline \multicolumn{7}{|l|}{$2013 / 2014$} \\
\hline Outubro & 17,50 & 13,50 & 22,10 & 404,00 & 214,70 & 12 \\
\hline Novembro & 20,50 & 16,90 & 24,90 & 473,00 & 136,30 & 11 \\
\hline Dezembro & 23,50 & 18,90 & 29,20 & 568,50 & 74,00 & 6 \\
\hline Janeiro & 24,80 & 20,60 & 30,50 & 475,60 & 182,60 & 17 \\
\hline Fevereiro & 24,10 & 20,90 & 29,00 & 434,10 & 220,60 & 16 \\
\hline Março & 21,10 & 17,20 & 26,20 & 386,00 & 146,30 & 11 \\
\hline Média & 21,92 & 18 & 26,98 & 456,87 & 162,42 & 12,17 \\
\hline \multicolumn{7}{|l|}{$2014 / 2015$} \\
\hline Outubro & 19,40 & 15,80 & 23,90 & 375,40 & 254,30 & 14 \\
\hline Novembro & 21,20 & 16,80 & 26,40 & 484,30 & 104,00 & 8 \\
\hline Dezembro & 22,80 & 18,90 & 27,90 & 478,10 & 138,20 & 12 \\
\hline Janeiro & 24,00 & 20,10 & 29,10 & 490,00 & 234,00 & 12 \\
\hline Fevereiro & 23,50 & 20,30 & 27,90 & 460,60 & 91,90 & 15 \\
\hline Março & 22,10 & 18,00 & 27,60 & 388,50 & 105,10 & 8 \\
\hline Média & 22,17 & 18,32 & 27,13 & 446,15 & 154,58 & 11,5 \\
\hline
\end{tabular}


Um dos principais fatores limitantes ao uso agrícola do solo Planossolo Háplico Eutrófico é a má drenagem (SANTOS et al., 2006). O excesso de umidade e os baixos índices de radiação podem ter influenciado a emissão de perfilhos e inflorescências. A disponibilidade de água às plantas é um dos fatores que mais influencia no crescimento e desenvolvimento das espécies. Paspalum notatum é uma espécie pioneira, desenvolvendo-se bem principalmente em solos mais secos e promovendo maior cobertura do solo, motivo que pode ter influenciado no resultado da produção potencial de semente dos acessos avaliados.

Embora o rendimento de sementes seja um traço complexo e afetado por práticas agrícolas, bem como fatores ambientais, os caracteres relacionados à produção de sementes revelam variação genética considerável, pré-requisito para melhoria por seleção direta ou indireta (BOELT e STUDER, 2010). Para a maior parte das características e ambientes avaliados, houve considerável variação.

Nas amostras coletadas na safra de 2013/2014 não ocorreu efeito de acessos para o número de inflorescências/ $\mathrm{m}^{2}$. O número de flósculos por inflorescência variou de 66,73 a 179,70, sendo que os acessos PN 09, e PN 10, ambos da espécie $P$. notatum, foram superiores aos demais, com número de flósculos de 179,70 e 169,10, respectivamente (Tabela 3). A produção potencial de sementes dos acessos analisados teve valores elevados, variando de 19152,00 a 135062,70 sementes $/ \mathrm{m}^{2}$, destacando-se o acesso PN 09 da espécie $P$. notatum, que teve como característica principal um maior número de flósculos por inflorescência. Esse potencial foi equivalente a 5,08 vezes a mais do que a produção potencial de sementes da testemunha gramabatatais, também um Paspalum notatum, mostrando variação importante dentro da mesma espécie.

Nas amostras coletadas no ano de 2014/2015 foi significativo o efeito de acessos para o número de inflorescências $/ \mathrm{m}^{2}$, variando de 89,50 a 793,50 (Tabela 4). Destacaram-se os acessos PN 11, PL 02 e PL 01, PL 03, PN 06 e PN 13, das espécies $P$. notatum, $P$. lividum, $P$. lepton, P. lepton, P. notatum, P. notatum, respectivamente. O número de flósculos por inflorescência variou de 77,50 a 173,40 , sendo que o acesso PN 10, da espécie $P$. notatum, apresentou o maior número. A produção potencial de sementes variou de 9973,75 a 81536,70 sementes $/ \mathrm{m}^{2}$, com destaque para o acesso PN 11 da espécie $P$. notatum. Esse potencial foi equivalente a 3,24 vezes a produção de sementes da testemunha grama-batatais, que obteve 25188,93 sementes $\mathrm{m}^{-2}$. $\mathrm{O}$ acesso com maior produção potencial de sementes encontra-se no grupo de acessos com maior número de inflorescências $\mathrm{m}^{-2}$.

O efeito do ano e sua interação com os genótipos é altamente variável entre as espécies e cultivares e resulta de uma complexa interação de fatores genéticos, fisiológicos e ambientais, tendo alguns dos componentes da produção de sementes altamente influenciados por fatores incontroláveis como ventos, chuvas, radiação solar, temperatura, umidade relativa do ar e fotoperíodo (SOUZA, 2013a). No presente trabalho, apesar da presença da interação genótipoambiente, alguns acessos permaneceram em posições semelhantes no ranking de produtividade potencial nas duas safras. Os acessos PN 11, PN 09, PN 10 e AP 01, das espécies $P$. notatum, $P$. notatum, $P$ notatum, Axonopus parodii, estiveram entre os seis melhores (terço superior) para a característica produção potencial de sementes e o PN 04, PN 14, PN 05 e a testemunha grama-batatais, ambos da espécie $P$. notatum, estiveram entre os seis piores (terço inferior), sugerindo possuir maior estabilidade que os demais. Estes acessos já foram avaliados em trabalhos anteriores como de interesse para compor gramados, com base em valor ornamental, velocidade de estabelecimento, baixa necessidade de manutenção, resistência à geada e competição com invasoras. Portanto, apresentam maior potencial de uso econômico em estratégias que utilizem a reprodução por sementes. Por serem os mais produtivos em uma das safras, permanecerem no terço superior na outra e superarem por larga margem a testemunha, os acessos PN 11 e PN 09, ambos da espécie Paspalum notatum, são os mais recomendados.

Além da produtividade potencial, é preciso avaliar nessas espécies a produtividade real de sementes dos acessos, uma vez que a ocorrência de cariopses ausentes é um dos grandes problemas relacionados à produção de sementes nos gêneros estudados, podendo estar associada a uma combinação de mecanismos selvagens de dispersão das sementes e sincronismo pobre das etapas do ciclo reprodutivo (SOUZA, 2013a).

\section{CONCLUSÕES}

Existe interação genótipo-ambiente para todas as características avaliadas. Entretanto, os acessos: PN 11, PN 09, PN 10 e AP 01, das espécies P. notatum e Axonopus parodii apresentam produção potencial de sementes consistentemente superior à testemunha grama-batatais. Para que estes acessos possam ser recomendados para a exploração na implantação de gramados por sementes ainda são necessários estudos quanto a produção efetiva de sementes, viabilidade e germinação.

\section{REFERÊNCIAS}

BATISTA, L.A.R.; GODOY, R. Capacidade de produção de sementes em acessos do gênero Paspalum. Revista Brasileira de Zootecnia, v.27, n.5, p.841-847, 1998.

BOELT, B.; STUDER B. Breeding for grass seed yield. In: BOLLER, B.; POSSELT, U.K.; VERONESI, F. Fodder crops and amenity grasses. New York: Springer, 2010. p.161-174.

BUSEY, P. Progress and benefits to humanity from breeding warm-season grasses for turf. In: SLEPER, D.A., ASAY, K.H., PEDERSON, J.F. Contributions from breeding forage and turf grasses. Atalta: Crop Science Society of America, 1989. p.49-70.

CARAMBULA, M. Producción de semillas de plantas forrajeras. Montevideo: Hemisferio Sur, 1981. 518p. 
CLAYTON, W.D.; RENVOIZE, S.A. Genera Graminum: grasses of the world. London: Royal Botanic Garden, 1986. (Kew Bulletin Additional Series, 13).

DUNCAN, R.R. Seashore Paspalum (Paspalum vaginatum Swartz). In: CASLER, M.D; DUNCAN, R.R. Turfgrass biology, genetics, and breeding. New Jersey: John Wiley, 2003.

GIRALDO-CAÑAS, D.; MAYORGA, C. Nuevos registros de gramíneas (Poaceae) para la flora de Colombia. Hickenia, v.28, p.99-103, 2001.

GIUSSANI, L.M.; COTA-SÁNCHEZ, J.H.; ZULOAGA, F.O.; KELLOG, E.A. A molecular phylogeny of the Grass subfamily Panicoideae (Poaceae) shows multiple origins of $\mathrm{C}_{4}$ photosynthesis. American Journal of Botany, v.88, p.1993-2012, 2001.

GOULD, F.W.; SHAW, R.B. Gramíneas: clasificación sistemática. México: AGT, 1992. 381p.

HICKENBICK, M.C.M.; VALLS, J.F.M.; SALZANO, F.M.; FERNANDES, M.I.B.M. Cytogenetic and evolutionary relationships in the genus Axonopus (Gramineae). Cytologia, v.40, p.185-204, 1975.

KISSMANN, K.G. Plantas infestantes e nocivas. 2.ed. São Paulo: Basf, Tomo I, 1999. p.824.

LÓPEZ, M.; GONZÁLEZ, M.C.; HERNÁNDEZ, L.; RAMIREZ, W.; MEDINA, R.; PÉREZ J.M. Estado del conocimiento del mejoramiento genético de cespitosas. Revista Pastos y Forrajes, v.36, n.3, p.278-287, 2013.

MOHRDIECK, K.H. Formações campestres do Rio Grande do Sul In: Campos nativos: melhoramento e manejo IV. Porto Alegre: Federacite, 1993. p.11-23.

MORRONE, O.; AAGESEN, L.; SCATAGLINI, M.A.; SALARIATO, D.L.; DENHAM, S.S.; CHEMISQUY, M.A.; SEDE, S.M.; GIUSSANI, L.M.; KELLOGG, E.A.; ZULOAGA, F.O. Phylogeny of the Paniceae (Poaceae: Panicoideae): integrating plastid DNA sequences and morphology into a new classification. Systematic Botany, v.37, n.3, p.671-376, 2012. DOI: <10.1111/j.1096$0031.2011 .00384 . \mathrm{x}>$
PÉREZ-ARBELÁEZ, E. Plantas útiles de Colombia. 4.ed. Bogotá: Litografía Arco. 1978. 831p.

PIZARRO, E.A. Potencial forrajero del género Paspalum. Pasturas Tropicales, v.22, n.1, p.38-45, 2000.

SANTOS, H.G.; JACOMINE, P.K.T.; ANJOS, L.H.C.; OLIVEIRA, V.A.; OLIVEIRA, J.B.; COELHO, M.R.; LUMBRERAS, J.F.; CUNHA, T.J.F. Sistema brasileiro de classificação de solos. 2. ed. Rio de Janeiro: Embrapa Solos, 2006. 306 p.

SOCIEDADE BRASILEIRA DE CIÊNCIA DO SOLO. COMISSÃO DE QUÍMICA E FERTILIDADE DO SOLO. Manual de adubação e calagem para os Estados do Rio Grande do Sul e Santa Catarina. $10^{\mathrm{a}}$ ed. Porto Alegre: SBCS, 2004.

SOUZA, F.H.D. Características morfoanatômicas associadas à produção de sementes em gramíneas. In: SOUZA, F.H.D.; MATA, F.P.; FÁVERO, A.P. Construção de ideótipos de gramíneas para usos diversos. Brasília: Embrapa, 2013a. p.61-80.

SOUZA, F.H.D. Construção de ideótipos: gramados. In: SOUZA, F.H.D.; MATA, F.P.; FÁVERO, A.P. Construção de ideótipos de gramíneas para usos diversos. Brasília: Embrapa, 2013b. p.213-226.

SOUZA, F.H.D. Os gramados e a forração vegetal permanente de superfícies de solo: exemplo de oportunidades perdidas? Embrapa Pecuária Sudeste, São Carlos-SP, 2013c. Disponível em: <http://ainfo. cnptia.embrapa.br/digital/bitstream/item/92404/1/CP FranciscoSouza.pdf $>$. Acesso em: 01 de julho de 2016.

ZULOAGA, F.O.; MORRONE, O. Revisión de las especies de Paspalum para América del Sur Austral (Argentina, Bolívia, Sur del Brasil, Chile, Paraguay y Uruguay). St. Louis: Missouri Botanical Garden Press, 2005. p.1-297. 